\title{
Augmented Microscope System for Training and Intra-Operative purposes
}

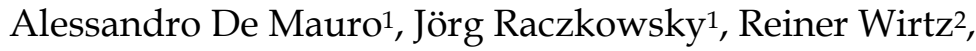 \\ Mark Eric Halatsch'2, Heinz Wörn ${ }^{1}$ \\ 1 Institute for Process Control and Robotics, University of Karlsruhe (TH) \\ ${ }^{2}$ University Hospital of Heidelberg \\ Germany
}

\section{Introduction}

In recent years, neurosurgery has been deeply influenced by new technologies. Computer Aided Surgery (CAS) offers several benefits for patients' safety but fine techniques targeted to obtain minimally invasive and traumatic treatments are required, since intra-operative false movements can be devastating, leaving patients dead. The precision of the surgical gesture is related both to accuracy of the available technological instruments and surgeon's experience. In this frame, medical training is particularly important. From a technological point of view the use of the Virtual Reality (VR) for the surgeons training and Augmented Reality (AR) for the intra-operative aid for treatments offer the best results. This paper presents a prototype for a mixed reality system for neurosurgical interventions embedded on a real surgical microscope for pre- and intra- operative purposes. Its main purposes are:

- realistic simulation (visual and haptic) of the spatula palpation of Low-Grade Glioma (LGG);

- $\quad$ stereoscopic visualization in AR of relevant 3D data for safe surgical movements in the image guided therapy (IGT) interventions.

This is the first prototype of a training system using a real microscope for neurosurgery.

\section{Motivation and Medical Background}

In neurosurgical operating theatre (OR) almost all interventions are carried out using a microscope (Fig.1). All the best commercial systems provide the surgeon with only a real two-dimensional overlay of the region of interest (e.g. tumour) inside the oculars of the operating microscope related on the preoperative processed patient's image data. The 3D environment reconstruction from 2D is another difficult and critical mental work for the surgeon. A first motivation to this work is related to the last considerations and the target is the improvements of a prototype of an AR stereoscopic microscope for neurosurgical interventions developed in our institute (Aschke et al., 1999) optimizing and completing the information set visible for the surgeons needs. The goal is to enhance the surgeon's ability 
for a better intra-operative orientation by giving him the three-dimensional view and other information he needs for a safe navigation inside the patients.

Another motivation is to enhance the current neurosurgical training in the specific frame of the realistic simulation (visual and haptic) of the spatula palpation of LGGs using a real surgical microscope. Traditional techniques for training in surgery include the use of animals, phantoms and cadavers. The main limitation of these approaches is that live tissue has different properties from dead tissue and also that animal anatomy is significantly different from the human. In other words, traditional surgical training is far from being realistic. Even if classical training is improved by the use of well illustrated books and excellent training movies recorded directly in the operating theatre, nowadays the main training for surgeons is still performed on the real patient. From several years simulation was validated by the scientific community and it was shown that VR simulators can speedup the learning process and improve the proficiency of surgeons prior to performing surgery on a real patient (Tomulescu \& Popescu, 1990). A comparison between computersimulation-based training and traditional mechanical simulator for basic laparoscopic skills, found that trainees who trained on the computer-based simulator performed better on subsequent porcine surgery (Youngblood et al. 2005).

From the medical point of view LGGs are intrinsic brain tumours that typically occur in younger adults. The objective of related treatment is to remove as much of the tumour as possible while minimizing damage to the normal brain. Pathological tissue may closely resemble normal brain parenchyma when looked at through the neurosurgical microscope. As a result, efforts to remove all tumour cells inevitably remove some of the normal brain and can leave behind small sections of tumours cells. Neuronavigation can help only partially because the brain-shift phenomena affects the pre-operative patient data after craniotomy and tissue removal. The tactile appreciation of the different consistency of the tumour compared to normal brain requires considerable experience on the part of the neurosurgeon and is a vital point.

Hardware (microscope, tracking system, tools) and software (navigation system based on the patient dataset) are both involved in the training and intra-operative activities. This consideration justifies the idea of a mixed reality system that uses similar environments and architecture setups for pre- and intra-operative use, providing a natural continuum between training system (based on VR) and intra-operative system (based on AR).

\section{Neurosurgical workflow}

In order to facilitate the understanding of this research study it is crucial to give a brief introduction to the steps involved before and during the neurosurgical procedures (workflow) and the related technology.

\subsection{Pre-operative phase}

The geometric models of the organs or the region of interest (e.g. tumour) are reconstructed from data acquired by CT, MRI or other means by a radiologist. In the intra-operative phase a tracking system is used to track the relative positions of the patient, relevant tools, and microscope. 


\subsection{Intra-operative phase}

In the OR, surgeon's eyes are typically on the microscope oculars but occasionally they need to see the screen in order to understand the correct position compared to the preoperative images (CT, MRI). All data are shown and navigated on the video using the normal three medical views (coronal, axial, sagittal) during the procedure. The position and orientation of an active tool tracked by the infrared tracking system and its relative position in the patient images are shown on the monitors. The two-dimensional contour of the region of interest is recognized as defined by the radiologist in the preoperative step. This two-dimensional shape is visible inside the commercial microscopes overlaid to the oculars views. The steps discussed here are shown in Fig.1

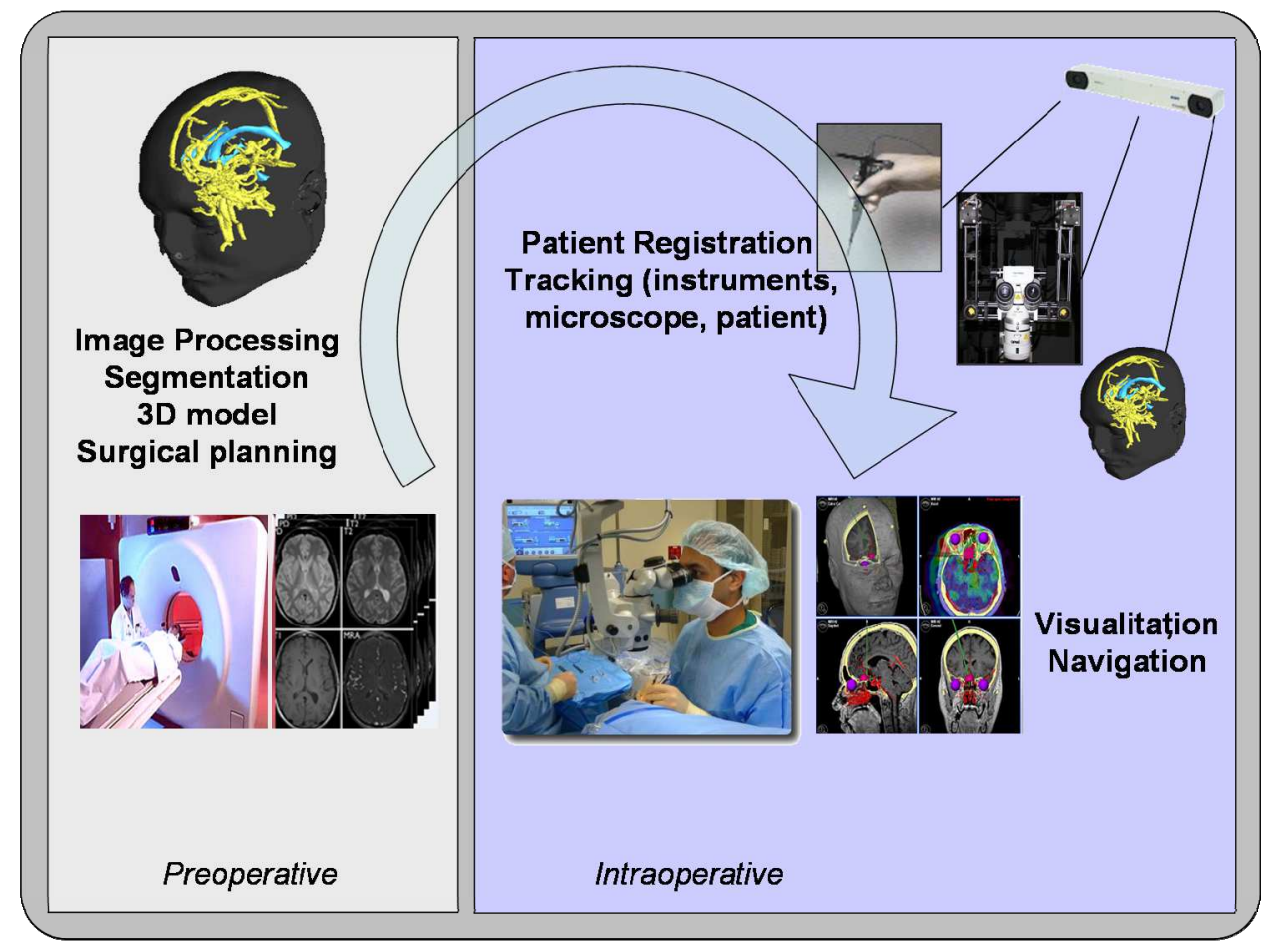

Fig. 1. Neurosurgery workflow

\section{State of art}

\subsection{State of art in VR training for Surgery}

The success of using haptic devices in medical training simulators has already been demonstrated by several commercial companies working in this field (i.e. Immersion Medical, Surgical Science, Mentice, and Reachin Technologies).

Other works show haptic simulation environment for laparoscopic cholecystectomy (Webster et al., 2003), for hysteroscopy (Montgomery et al., 2001) , interventional cardiology 
procedures, incorporating blood flow models and models of cardiopulmonary physiology (Cotin et al., 2000), common bile duct exploration (Basdogan et al., 2001).

On the other hand, the state-of-the-art in neurosurgical simulation (Goh et al., 2005) shows only a few examples of VR based systems which use force feedback (Luciano et al., 2008, Sat o et al., 2006 and Wiet et al., 2000). Because a neurosurgical microscope is used in a large percentage of interventions, the realism of these simulators is limited by the architecture: they use either standard monitors or head mounted displays but not a real surgical microscope.

\subsection{State of art in AR for Neurosurgery}

The best commercial systems (i.e. Brainlab and Stryker) provide the neurosurgeons with only a real two-dimensional overlay of the region of interest (ex. tumour) inside the oculars of the operating microscope related on the preoperative processed patient's image data. The three-dimensional environment reconstruction from $2 \mathrm{D}$ is another difficult and critical mental work for the surgeon. There were only two working examples of AR 3D stereoscopic microscope for neurosurgery. The first was described in (Edwards, P. et al., 2000) and the second was developed in our laboratories (Aschke et al. 1999). We improved this previous work extending it with a higher level graphic modality and enhancing its realtime performances.

\section{Methods}

\subsection{Virtual reality training system}

In neurosurgical interventions monitors and operating microscopes are both commonly employed. In order to understand the correct tumour position compared to the preoperative images (CT, MRI) surgeon's eyes are normally on the microscope oculars and only occasionally glance at a larger screen. This second view is crucial in image guided therapy (IGT) to check the correct position of the surgical tools inside the patient brain.

A complete simulation system for neurosurgical training requires:

- $\quad$ simulation of the virtual patient inside the real microscope oculars;

- $\quad$ force feedback rendering directly at the user's hand;

- common navigation software used in OR. 


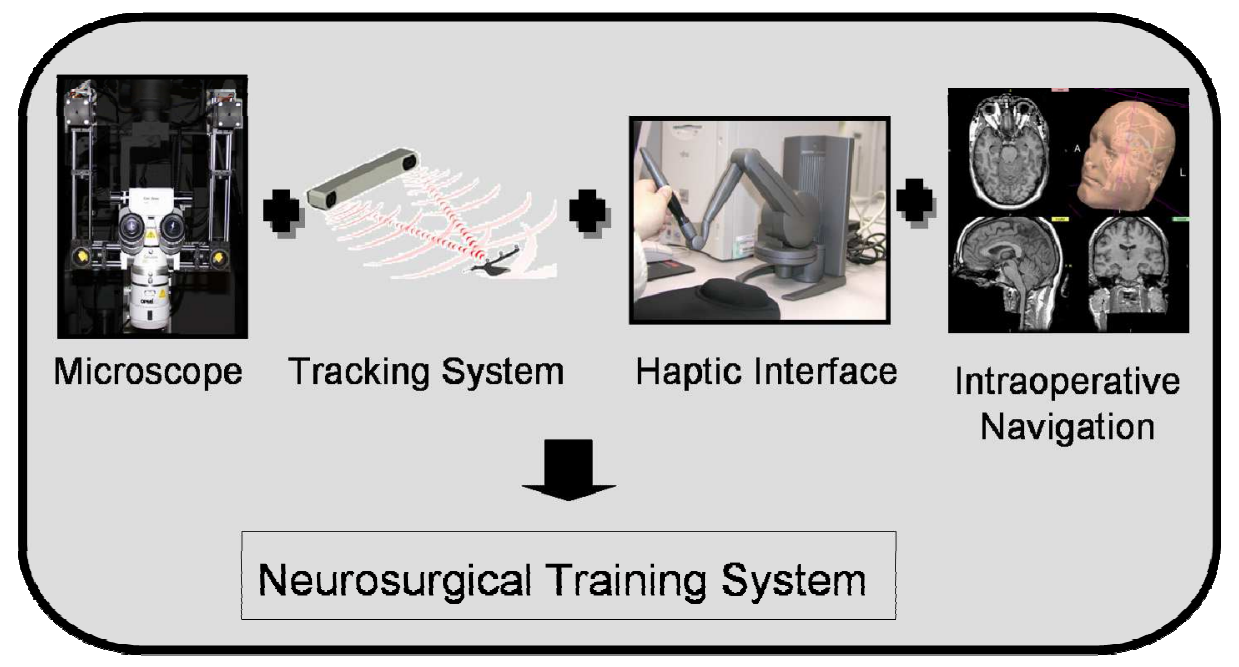

Fig. 2. Neurosurgical training system architecture

A virtual environment is geometrically built using real patients' data affected by a LG from the standard medical imaging devices. During this process human organs are accurately reconstructed from real patient images using the open source software 3DSlicer.

Region growing algorithm has been used for segmentation, and next organ and region of interests are classified. The 3D triangularized surface model of the organ is obtained with a Marching cubes strategy and it's imported directly into our application, refined and converted from the VTK file format (3DSlicer output) to X3D. The 3D environment description language used is $\mathrm{X} 3 \mathrm{D}$ because it is the natural successor of the well known VRML. It means that the complete scene in the application can be rendered in a web browser for future extension as web- collaborative and distributed training. Texturing is applied at this step in order to improve realism.

The registration step between these two virtual environments is carried out applying the right transformation matrices. To obtain the 3D models of the surgical tools we use a Laser ScanArm (FARO) as a rapid and accurate prototyping tool.

The visual output is provided by the rendering software developed in $\mathrm{C}++$ and built on the open source GPU licensed and cross-platform H3D, a scene-graph API based on OpenGL for graphics rendering.

Collision detection module (requested to compute collision response) is based on based on model partition (Van den Bergen, C., 2004), the strategy of subdividing a set of objects into geometrically coherent subsets and computing a bounding volume for each subset of objects. It's connected to the physical model for the simulation of tool interactions and brain deformations and it's developed in OpenGL to obtain high level performance and realism. The physical modelling method is based on Mass-Spring-Damper (MSD) and consists of a mesh of point masses connected by elastic links and mapped onto the geometric representation of the virtual object. This method is employed in our prototype to describe the mechanical properties of the virtual bodies computing the force feedback to the haptics and the organ deformations to be visualised. It is a discrete method characterized by low computable load, simplicity, low accuracy and low risk of instability because it uses Newton 
dynamics to modify the point-masses positions and creates deformations with consideration to volume conservation. Brain tissue properties are modelled with MSD upon the OpenHaptics library (Sensable Tech.). The tissue parameters (stiffness, damping, friction, etc.) were evaluated together with our medical partner (Department of Neurosurgery, University Hospital of Heidelberg) using different training sections and processing empiric data.

Different haptic renderings were tested for a better optimization of the deformations. In order to have a complete training platform a video navigation system containing different $3 \mathrm{D}$ volume views is required. To achieve this, we have connected our system with the image guided therapy module of 3DSlicer. A haptic device (Phantom Desktop) provides the surgeon with an immersive experience during the interaction between the surgical tools and the brain or skull structures of the virtual patients. Its force feedback workspace and other important properties (nominal position resolution and stiffness range) make it suitable to be used in an ergonomic manner in conjunction with the microscope.

The 3D real-time position of the two viewpoints (one of each microscope ocular) is determined in the virtual environment through the use of passive markers affixed to the microscope and tracked by the infrared optical tracker (Polaris NDI). In order to speed up the simulation we modified the original library for tracking developed in our laboratories. In this way, data collected from Polaris is sent using the local area network to several given IP addresses and ports using the open-source OpenIGTLink of 3DSlicer modified for our needs. This allows a distributed architecture with the separation between rendering (graphical and haptic) and tracking PC with advantages in terms of computational load and realism (the average frame rate for graphical rendering is $31 \mathrm{fps}$ and for the haptic $998 \mathrm{fps}$ ). The collisions between organs and surgical tools produce forces which have to be replicated by the haptic interface and organ deformations, which have to be graphically rendered.

The main operating task simulated is the visual and tactile sensations of brain palpation (healthy or affected by LGG) pushing aside the human tissue using a neurosurgical spatula.

\section{Augmented Reality extensions}

The architecture described can be adapted for intra-operative purposes. In this instance, a surgeon needs the basic setup for the IGT interventions: microscope, monitors and surgical tools. The same virtual environment can be AR rendered onto the microscope optics with the difference that now the complete anatomy is considered rigid (deformable organs are not requested in this frame since only geometrical information are required) as well as the haptic interface replaced by new navigated infrared active tools.

The prototype is capable of tracking, in real time, the microscope, the patient's head and one or more surgical tool (pointer with active markers).

Fig. 3 shows the AR views inside the microscope oculars in which is possible identify the 3D region of interest (in this example the brain surface and craniotomy area is rendered). The microscope hardware related part was realized at our institute, as mentioned before and described in a previous work (Aschke et al. 1999). Registration with ICP (P.J. Besl \& N.D. McKay, 1992) and camera calibration are carried out with similar procedure adopted in the previous prototype. Both are off-line steps and required for a perfect alignment between real and virtual world at the microscope view. 


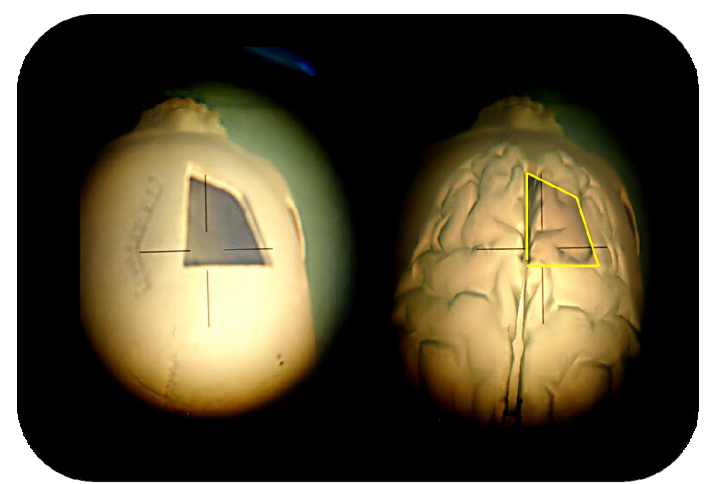

Fig. 3. AR microscope. Left: skull. Right: 3D model of the brain superimposed on the skull.

\section{Conclusion}

This paper presents the development of the first mixed reality system for training and intraoperative purposes in neurosurgery embedded on a real microscope. The main training task is the advanced simulation of brain tissue palpation enabling the surgeon to distinguish between normal and affected with a LGG brain tissue. Force feedback interaction with soft and hard tissues is made available to the surgeon in order to provide a complete immersive experience. The second feature allows the system to be employed as an AR microscope inside the OR. In this case a complex 3D environment is rendered by a stereoscopic image injection directly inside the microscope oculars for a better real time brain navigation and space cognition. 3DSlicer is, in both previous functional modalities, directly connected to the NDI tracking system in order to provide the navigation inside the real patient's images on the screen. The previously described software architecture guarantees performances and portability.

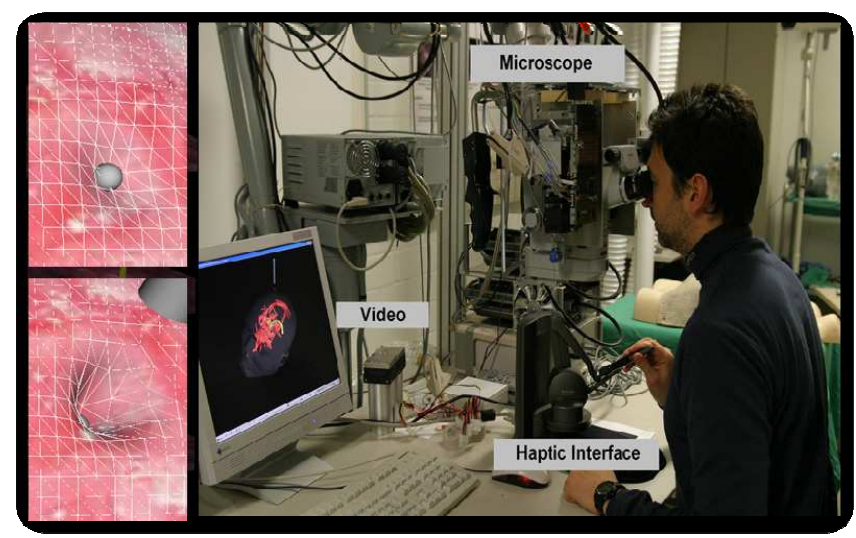

Fig. 4. Simulator. Left: brain tissue deformations. Right: complete prototype. 


\section{Acknowledgments}

This research is a part of the European project "CompuSurge" funded by FP7 EST "Marie Curie" research network.

\section{References}

3DSlicer, URL: http:/ / www.slicer.org/ [status January 2008]

Aschke, M. et al. (2003) Augmented reality in operating microscopes for neurosurgical interventions. Wolf and Strock editors, Proceedings of 1st International IEEE EMBS Conference on Neural Engineering, pp. 652--655

Besl, P.J. \& McKay, N.D. (1992), A Method for Registration of 3-D Shapes, IEEE Trans. Pattern Anal. Mach. Intell., vol. 14, 1992, pp. 239-256.

Basdogan, C., et al. (2001) Virtual Environments for Medical Training: Graphical and Haptic Simulation of Common Bile Duct Exploration. IEEE-ASME Trans. Mechatronics, vol. 6, no. 3, pp. $26-285$.

Cotin S. et al. (2000) An Interventional Cardiology Training System. Proceedings of Medicine Meets Virtual Reality, pp. 59--65

Edwards, P. et al., (2000), Design and evaluation of a system for microscope-assisted guided interventions (MAGI), Medical Imaging, IEEE Transactions on, vol. 19, pp. 10821093.

Goh, K.Y.C. et al. (2005) Virtual reality application in neurosurgery. Proceedings of the 2005 IEEE, Engineering in Medicine and Biology 27th Annual Conference, Shanghai

H3D, URL: http://www.h3dapi.org/ [status January 2008]

Luciano, C. et al. (2008) : Second generation haptic ventriculostomy simulator using the immersivetouch ${ }^{\mathrm{TM}}$ system. Proceedings of Medicine Meets Virtual Reality 14, Long Beach, CA, USA.

Montgomery, K. et al.(2001), Surgical simulator for operative hysteroscopy, IEEE Visualization 2001, pp. 14-17.

OpenHaptics, URL: http://www.sensable.com/products-openhaptics-toolkit.htm [status March 2009].

Sato, D. et al. (2006), Soft tissue pushing operation using a haptic interface for simulation of brain tumor resection. Journal of robotics and mechatronics, vol. 18, pp. 634-642.

Tomulescu, V \& Popescu, I. (1990) The use of LapSim virtual reality simulator in the evaluation of laparoscopic surgery skill. Preliminary result, Chirurgia (Bucharest, Romania), vol. 99, pagg. 523-7.

Van den Bergen, C. (2004), Collision detection in interactive 3D environment. Elsevier Morgan Kaufmann, S.Francisco, USA, 2004

Webster, R. et al. (2003) A haptic surgical simulator for laparoscopic cholecystectomy using real-time deformable organs. Proceedings IASTED International Conference on Biomedical Engineering, June 25-27, Salzburg, Austria.

Wiet, G. et al. (2000), Virtual temporal bone dissection simulation, Proceedings of Medicine Meets Virtual Reality 2000, pp. 378--384, J. D. Westwood, Ed., Amsterdam, The Netherlands.

Youngblood, P.L. et al. (2005) Comparison of training on two laparoscopic simulators and assessment of skills transfer to surgical performance, Journal of the American College of Surgeons, vol. 200, pp. 546-551. 


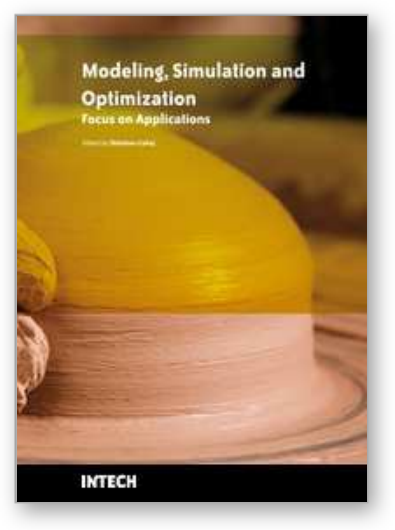

\author{
Modeling Simulation and Optimization - Focus on Applications \\ Edited by Shkelzen Cakaj
}

ISBN 978-953-307-055-1

Hard cover, 312 pages

Publisher InTech

Published online 01, March, 2010

Published in print edition March, 2010

The book presents a collection of chapters dealing with a wide selection of topics concerning different applications of modeling. It includes modeling, simulation and optimization applications in the areas of medical care systems, genetics, business, ethics and linguistics, applying very sophisticated methods. Algorithms, 3-D modeling, virtual reality, multi objective optimization, finite element methods, multi agent model simulation, system dynamics simulation, hierarchical Petri Net model and two level formalism modeling are tools and methods employed in these papers.

\title{
How to reference
}

In order to correctly reference this scholarly work, feel free to copy and paste the following:

Alessandro De Mauro, Jorg Raczkowsky, Reiner Wirtz, Mark Eric Halatsch, Heinz Worn (2010). Augmented Microscope System for Training and Intra-Operative purposes, Modeling Simulation and Optimization - Focus on Applications, Shkelzen Cakaj (Ed.), ISBN: 978-953-307-055-1, InTech, Available from:

http://www.intechopen.com/books/modeling-simulation-and-optimization-focus-on-applications/augmentedmicroscope-system-for-training-and-intra-operative-purposes

\section{INTECH}

open science | open minds

\section{InTech Europe}

University Campus STeP Ri

Slavka Krautzeka 83/A

51000 Rijeka, Croatia

Phone: +385 (51) 770447

Fax: +385 (51) 686166

www.intechopen.com

\section{InTech China}

Unit 405, Office Block, Hotel Equatorial Shanghai

No.65, Yan An Road (West), Shanghai, 200040, China

中国上海市延安西路65号上海国际贵都大饭店办公楼 405 单元

Phone: +86-21-62489820

Fax: $+86-21-62489821$ 
(C) 2010 The Author(s). Licensee IntechOpen. This chapter is distributed under the terms of the Creative Commons Attribution-NonCommercialShareAlike-3.0 License, which permits use, distribution and reproduction for non-commercial purposes, provided the original is properly cited and derivative works building on this content are distributed under the same license. 